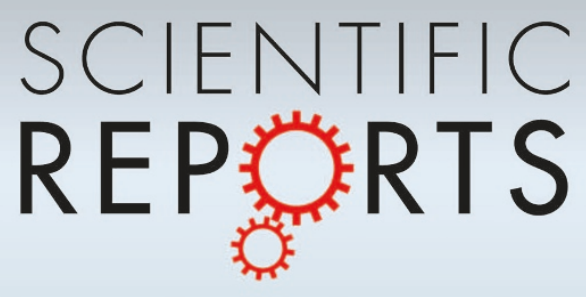

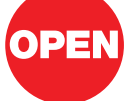

SUBJECT AREAS:

STRUCTURE OF SOLIDS

AND LIQUIDS

METALS AND ALLOYS

MECHANICAL PROPERTIES

MINERALOGY

Received

4 October 2012

Accepted

11 December 2012

Published

18 January 2013

Correspondence and requests for materials should be addressed to

M.L.S. (mlsui@bjut. edu.cn)

\title{
Microstructural fingerprints of phase transitions in shock-loaded iron
}

\author{
S. J. Wang' ${ }^{1}$, M. L. Sui ${ }^{2}$, Y. T. Chen ${ }^{3}$, Q. H. Lu', E. $\mathrm{Ma}^{4}$, X. Y. Pei ${ }^{3}$, Q. Z. Li ${ }^{3}$ \& H. B. $\mathrm{Hu}^{3}$
}

'Shenyang National Laboratory for Materials Science, Institute of Metal Research, Chinese Academy of Sciences, Shenyang 110016 , China, ${ }^{2}$ Institute of Microstructure and Property of Advanced Materials, Beijing University of Technology, Beijing 100124, China, ${ }^{3}$ Institute of Fluid Physics, China Academy of Engineering Physics, Mianyang 621900, China, ${ }^{4}$ Department of Materials Science and Engineering, Johns Hopkins University, Baltimore, Maryland 21218, USA.

The complex structural transformation in crystals under static pressure or shock loading has been a subject of long-standing interest to materials scientists and physicists. The polymorphic transformation is of particular importance for iron $(\mathrm{Fe})$, due to its technological and sociological significance in the development of human civilization, as well as its prominent presence in the earth's core. The martensitic transformation $\alpha \rightarrow \varepsilon(b c c \rightarrow h c p)$ in iron under shock-loading, due to its reversible and transient nature, requires non-trivial detective work to uncover its occurrence. Here we reveal refined microstructural fingerprints, needle-like colonies and three sets of $\{112\}<111>$ twins with a threefold symmetry, with tell-tale features that are indicative of two sequential martensitic transformations in the reversible $\alpha \rightarrow \varepsilon$ phase transition, even though no $\varepsilon$ is retained in the post-shock samples. The signature orientation relationships are consistent with previously-proposed transformation mechanisms, and the unique microstructural fingerprints enable a quantitative assessment of the volume fraction transformed.

$\mathrm{t}$ ambient conditions, Fe is stable in its $\alpha$-phase with the body-centered-cubic (bcc) crystal structure. At high pressures, Fe takes on the $\varepsilon$ form, switching to the hexagonal-closed-packed (hcp) lattice. While the phase diagram of $\mathrm{Fe}$ under hydrostatic pressure is well established ${ }^{1}$, it is much more challenging to ascertain what happens in Fe when it is subjected to shock-loading. Understanding dynamic phase evolution is critically relevant to many applications of iron and steels under explosion and shock processing conditions.

Nearly sixty years ago, an $\alpha \rightarrow \varepsilon$ martensitic transformation (MT) was postulated for Fe upon shock-loading (to $13 \mathrm{GPa})^{2,3}$. But the confirmation of this shock-induced transformation requires non-trivial detective work, because the high-pressure $\varepsilon$-phase induced during the loading transient transforms back into the $\alpha$-phase immediately after the shock is over. As a result, the reversible $\alpha \rightarrow \varepsilon$ transformation leaves no $\varepsilon$-phase behind, for direct observation in the microstructure of postmortem samples. Earlier experiments had to rely on indirect evidence such as wave profile measurements $s^{2-7}$ : the stable three-wave shock fronts of the wave profiles measured during shock-loading suggested a polymorphic phase transition. More recent efforts succeeded in acquiring diffraction patterns from the $\varepsilon$-phase via in-situ x-ray diffraction both in single crystal and polycrystal ${ }^{8-10}$, and captured signatures from in-situ extended $\mathrm{x}$-ray absorption fine structure ${ }^{11}$ in laser shocked Fe. These experimental findings are consistent with the predicted $\alpha \rightarrow \varepsilon$ transformation in recent multi-million-atom moleculardynamics simulations ${ }^{9,12-15}$. However, key questions remain open in this area: 1) While in-situ X-ray diffraction measurements provided information about the atomic pathway of the phase transition, and the width of diffraction peaks could be used to deduce the microstructural scale involved, the previous experiments, including those that examined recovered samples after shock ${ }^{16,17}$, were not able to directly image the morphological features (such as size, shape and density of domains, defects, twins, interphase boundaries, etc.) that are characteristic or indicative of the $\alpha \rightarrow \varepsilon$ transition. 2) Without such microstructural information, the experiments so far cannot quantitatively assess the volume fraction actually transformed to the $\varepsilon$-phase, in different locations of the shocked sample, especially when the loading geometry/condition is complex and the sample is of bulk form. In recovered samples, only a minute amount of $\varepsilon$-phase could be retained by rapid quenching using a femto-second laser ${ }^{18}$.

The present work demonstrates that insight into these issues can be obtained by performing ex-situ transmission electron microscopy (TEM) experiments, on recovered samples of shock-compressed Fe. In particular, we have detected under TEM signature microstructures retained in the post-shock sample, including needle-like colonies arising from shock-induced $\alpha \rightarrow \varepsilon$ transition and three sets of ultrafine twinned $\alpha$ domains inside the colonies which originate from the three-fold prismatic planes of the $\varepsilon$ phase during the $\varepsilon \rightarrow \alpha$ reverse transition upon unloading. These microstructural fingerprints can be used to infer that the $\alpha \rightarrow \varepsilon \rightarrow \alpha$ MTs have indeed 

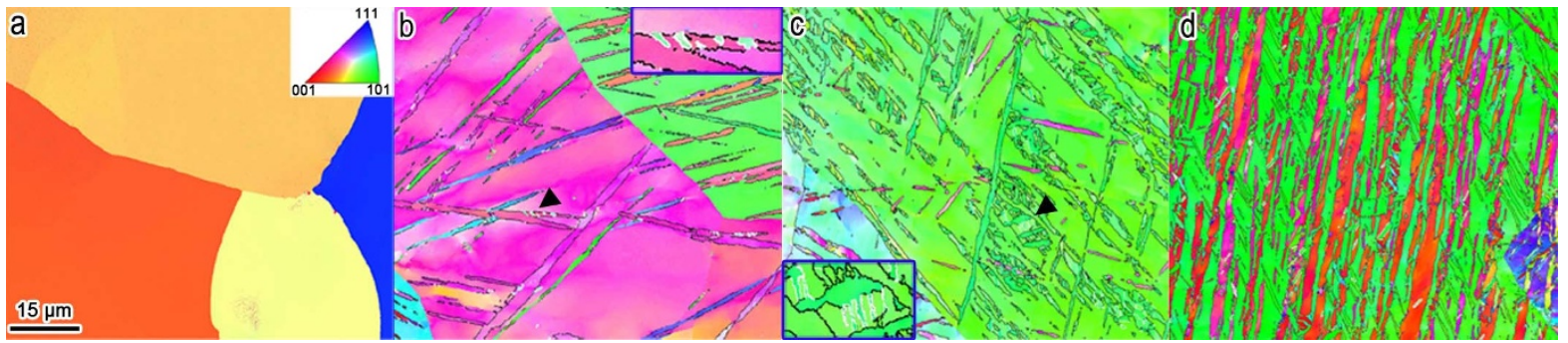

Figure 1 Inverse pole figure color map overlapped with twin boundaries in $\boldsymbol{\alpha}$-Fe. (a) before and (b,c,d) after shock-loading. Many twin lamellae appear after shock-loading. The $\{112\}<111>\left(\sum 3\right)$ twins with boundaries delineated by black lines are dominant, while the $\{332\}<113>$ twins (white lines) are very limited. The colors in the IPF map correspond to the crystallographic orientation normal to the observed plane, indicated in the stereographic triangle superimposed in (a). The insets in $(\mathrm{b}, \mathrm{c})$ are the enlarged images of the corresponding regions marked by arrows.

occurred in the shocked material, with unique features that are in line with specific mechanisms proposed for both the forward and reverse $\alpha-\varepsilon$ MTs. More importantly, based on the postmortem microstructures, we can assess quantitatively the volume percentage transformed under different shock-loading conditions and in different regions of the sample volume. This investigation thus provides quantitative information that is essential to the development and validation of physical models and computational algorithms (such as macroscopic treatment via numerical calculations) about shockinduced phase transformations.

\section{Results}

Bulk polycrystalline Fe with a hollow cylindrical shape was shocked by explosive loading on outside surface (see the details of experimental setup in the Methods section). The peak pressure near the loading surface has been estimated as $\sim 16.4 \mathrm{GPa}$ (higher than the pressure threshold anticipated for the $\left.\alpha \rightarrow \varepsilon \mathrm{MT}^{2,3}\right)$. The original sample consists of equi-axed $\alpha$-Fe grains with sizes in the range of tens of micrometers, as indicated by the electron backscatter diffraction (EBSD) orientation map in Fig. 1a. Fig. 1 shows inverse pole figure (IPF) color map overlapped with twin boundaries (black and white lines). The colors in the IPF map correspond to the crystallographic orientation normal to the observed plane, indicated in the stereographic triangle superimposed in Fig. 1a. After shock-loading from the cylinder outside surface, fine sub-grain features can be observed in the original $\alpha$-Fe grains, as seen in the IPF color maps in Fig. 1, as well as in the TEM micrographs in Fig. 2. In what follows, these microstructural details will be shown to be revealing regarding phase transitions, while X-ray diffraction merely confirmed that $\alpha$-Fe remained the only phase present after the shock throughout the sample, with no indication of phase transformation.

Specifically, Figs. 1(b-d), taken at locations about $4.6 \mathrm{~mm}$ (local pressure is calculated as $\sim 12.7 \mathrm{GPa}$ by hydrodynamic code shown in the Methods section), $1.9 \mathrm{~mm}(\sim 13.3 \mathrm{GPa})$ and $1.2 \mathrm{~mm}(\sim 14.2 \mathrm{GPa})$ inward from the loading surface, respectively, show ultrafine lamellar structures. Based on the orientation information revealed by EBSD orientation map, these planar defects are twins and their boundaries; see TEM details later. There are more and finer twin lamellae near the cylinder outside surface [Figs. $1(\mathrm{c}, \mathrm{d})$ ] than that much further away from the surface (Fig. 1b).

The twinned structures near the outside surface have been investigated carefully under TEM. Fig. 2a is a montage collection of 18 bright-field TEM images, taken one by one from an area of about $35 \times 35 \mu^{2}$ (extracted from one large original $\alpha$-Fe grain in Supplementary Fig. S1) viewed along the $\langle 110\rangle$ zone axis. In this large-area TEM view, in addition to some dislocation/strain contrast, there are two levels of salient microstructures that will be discussed in detail below. On the finest level, there are numerous fine lamellar structures and boundaries, which are the twin lamella features mentioned earlier (see EBSD findings in Fig. 1). A close-up view is shown in the high-magnification TEM micrograph in Fig. 2b, which

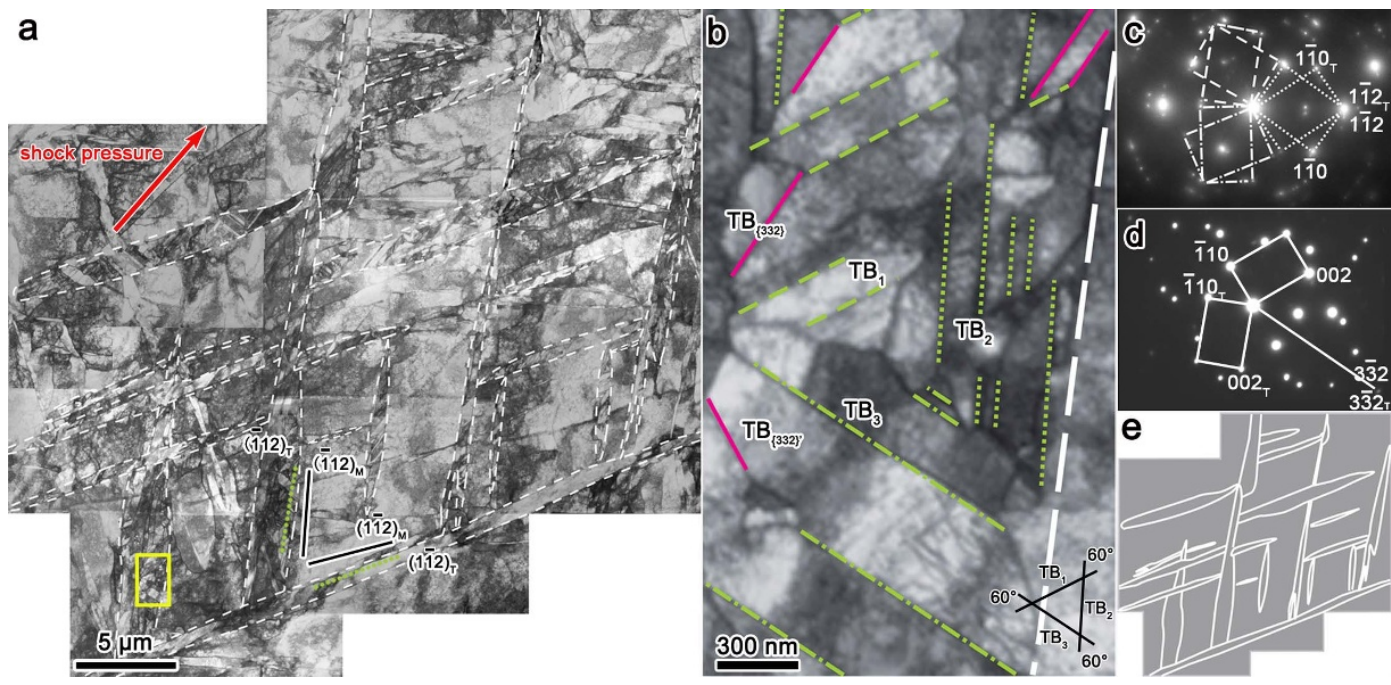

Figure $2 \mid$ Microstructural fingerprints of phase transitions in shock-loaded iron. (a) TEM micrograph showing the microstructure of shockcompressed Fe. (b) Enlarged view of the yellow rectangle area in a): $\mathrm{TB}_{1}, \mathrm{~TB}_{2}$ and $\mathrm{TB}_{3}$ (dashed, dotted, and dash-dot green lines) stands for three sets of $\{112\}$ twins; $\mathrm{T}_{332}$ and $\mathrm{T}_{(332)}$ ' (red solid lines) for two sets of $\{332\}$ twins. The triangle with black solid lines shows the included angle of the three sets of $\{112\}_{\text {bcc }}$ twins. (c) SAED of the three sets of $\{112\}$ twins. (d) SAED of $\mathrm{T}_{332}$. (e) Contours of the boundaries of needle-type-martensite-like regions in a) (white dashed lines). 
enlarges the yellow boxed region in Fig. 2a. One observes three sets of straight edge-on boundaries (marked with green lines) of intersecting lamellar bands. These, as seen from the selected area electron diffraction (SAED) patterns in Fig. $2 c$, are three sets of $\{112\}<111>$ twins with a threefold symmetry. In addition, Fig. $2 d$ gives the SAED pattern indicating the $\{332\}<113>$ twin relationship for the boundaries (red solid lines) at the upper right corner of Fig. 2b. These $\{332\}<113>$ twins coexist with the dominant $\{112\}<111>$ twins. But since they also exit in plastically deformed regions far away from the loading surface (Fig. 1b), they cannot be taken as a signature indicative of phase transformation ${ }^{17}$.

At a coarser level, a salient microstructure in Fig. 2a is the presence of many needle-like regions (for the clear boundaries between colonies and matrix, see the original TEM image in Fig. S2), enclosed by white dashed lines. The needle-like colonies form networks, dividing the original single $\alpha$-grain into many blocks. A cartoon delineating the network is displayed in Fig. 2e. The multiple sets of twin lamellae discussed above are contained inside these needle-like regions.

To recapitulate, both before and after shock-loading the Fe is in its $\alpha$-phase. But a hierarchical microstructure composed of two levels/ types of unusual features has been detected after the shock. These salient features are the fingerprints that memorize and reflect the (phase transformation) history the material has been through. In the following, we explain the origins of these features and associate them with the phase transformations experienced.

We first discuss the edge-on boundaries (marked with three types of green lines in Fig. 2b). As shown above, these are three sets of lamellae of $\{112\}$ twins, in three different orientations. As seen in the micrograph, they form an acute angle of $60^{\circ}$ in between when they intersect (inset of Fig. 2b). To explain this and relate it to the $\alpha \rightarrow \varepsilon \rightarrow \alpha$ MTs, we first point out that when viewed along the $<110>$ zone axis of the bcc $\alpha$-Fe, two $\{112\}$ planes should have formed an inclusion angle of $70.5^{\circ}$ or $109.5^{\circ}$, rather than $60^{\circ}$ or $120^{\circ}$. Therefore, the three sets of $60^{\circ}\{112\}$ planes in Fig. $2 b$ are not $\{112\}$ twins of $\alpha$-Fe within a single crystal (grain). In the meantime, we note that martensite structures are well known to often comprise a twinned microstructure $^{19}$, as it helps to relieve the strains that accompany the MT. The three sets of twinned lamellae are thus three variants of a martensite, forming three domains that later impinge and intersect. In other words, each set of the $\{112\}$ lamellar twins arises and grows out of a separate domain (subgrain); and when two neighboring sets impinge, they meet at the $60^{\circ}$ angle.

What, then, is the origin of the $60^{\circ}$ orientational relationship and threefold symmetry (Fig. 2b)? The answer is that the martensite formation is not from the bcc $\alpha$-phase but rather from an intermediate phase, which has an intrinsic three-fold symmetry. In other words, before the MT mediated by twinned structures, the region in question (the needle-like colony) should have already undergone the $\alpha \rightarrow \varepsilon$ transformation. In the hcp $\varepsilon$-phase, MT habit plane is expected to be $\{1-100\}_{\text {hcp }}$ which has six-fold symmetry ${ }^{20}$. There are three crystallographically equivalent $\{1-100\}$ planes, and this threefold symmetry is retained in the reverse transformation back to the $\alpha$-phase.

To lend support to this assertion, we note that Zhang et al. ${ }^{20}$ have observed earlier a similar microstructural feature for $\alpha$-Ti(hcp) $\rightarrow \beta$ $\mathrm{Ti}$ (bcc) MT induced by rapid heating: three sets of edge-on $\{112\}$ martensite twin lamellae of $\beta$ - $\mathrm{Ti}(\mathrm{bcc})$ with an angle of $120^{\circ}$ (or $60^{\circ}$ ), surrounding an untransformed $\alpha-\operatorname{Ti}(\mathrm{hcp})$ region in a triangle shape. The orientation relationship between $\alpha$-Ti and $\beta$-Ti is determined to be $(1-100)_{\alpha-\mathrm{Ti}} / /(-112)_{\beta-\mathrm{Ti}}$ (and $\left.[0001]_{\alpha-\mathrm{Ti}} / /[110]_{\beta-\mathrm{Ti}}\right)$. Similar to these previous findings, the three sets of $\{112\}_{\alpha-\mathrm{Fe}}$ lamellar twins in Fig. $2 b$ should be the $\alpha$-Fe martensite variants (lamellar twins) originated from three different $\{1-100\}_{\varepsilon-F e}$ planes, and the angle of $60^{\circ}$ is inherited from the three-fold symmetry characteristic of the $\{1-100\}_{\varepsilon-F e}$ planes. Each of the three variants grows out of one prismatic $\{1-100\}$ plane of the $\varepsilon$-phase, thus meeting with $60^{\circ}$ angles.
The microstructure observed is therefore a signature revealing the $\alpha \rightarrow \varepsilon \rightarrow \alpha$ transformation sequence. Shock-loading does induce the $\alpha \rightarrow \varepsilon$ transition, and the intermediate $\varepsilon$-phase transforms back to $\alpha$ phase during the ensuing unloading. A similar argument (see the Discussion section) can easily exclude the involvement of an intermediate face-centered-cubic $\gamma$-phase ${ }^{13,21}$ in the MTs.

We next discuss the networked needle-like regions (outlined by white dashed lines in Fig. 2a). If the bundles of the twin lamellae contained inside the needles come from the $\varepsilon \rightarrow \alpha$ reverse transition as discussed above, then the needle-type regions themselves would be the locations that have experienced the $\alpha \rightarrow \varepsilon$ forward MT. This belief is supported by the morphology: the needle-like shape closely resembles that known for a martensite colony ${ }^{19}$. Indeed, outside of the needle-like regions, all the blocks have the same SAED pattern and they apparently belong to one matrix grain and retain the original orientation.

Another supporting evidence is the character of the boundary between the needle colonies and the matrix. These needles run in two different orientations in this original $\alpha$-grain (Fig. 2e). For each needle, the boundaries are comprised of some straight lines and steps. The straight parts are edge-on $\{112\}_{\mathrm{bcc}}$ twin boundaries, and on the needle (twin) side the $\{112\}_{\mathrm{T}}$ plane (the green dotted line in Fig. 2a) is parallel to the boundary. But on the matrix side the $\{112\}_{M}$ plane (the black solid line in Fig. $2 \mathrm{a}$ ) has a small tilt angle $(\varphi)$ of $\sim 5^{\circ}$ inclined with respect to the boundary. The $\{112\}$ boundary itself is thus not a coherent twin boundary. These features are clearly seen in Fig. 3, from the high-resolution TEM image and the small angle between the $\{112\}$ diffraction spots from the twin and the matrix in the corresponding SAED pattern in the inset. This character $\left(\varphi \approx 5^{\circ}\right)$ at the boundary is in fact expected from the compressionshuffle mechanism mediating the $\alpha \rightarrow \varepsilon$ transformation ${ }^{9}$ under shock-loading (different from the hydrostatic pressure case ${ }^{22}$ ), as schematically illustrated in Fig. 4a. A uniaxial loading compresses the atoms in the $(110)_{\text {bcc }}$ plane along the $[001]_{b c c}$ direction by $18.4 \%$ with respect to the uncompressed bcc state (top in Fig. 4a) to form a hexagon. Then every other $(110)_{\text {bcc }}$ plane shuffles by $a_{0} /(3 \sqrt{2})$ toward the $[-110]$ direction (small arrows) to form an hcp structure (bottom). The resulting $\varphi$ between the resulting $(1-100)_{\text {hcp }}$ plane and the original $(-112)_{\text {bcc }}$ plane is $5.3^{\circ}$. We indeed observe $\varphi=5.2 \pm 0.7^{\circ}$ statistically (for the statistical details, see Supplementary Table S1) between the $(-112)_{b c c}$ (as $\{112\}_{M}$ in the original $\alpha$-phase in Fig. 2a) and the (1-100) $)_{\text {hcp }}$ in the $\varepsilon$-phase (as $\{112\}_{\mathrm{T}}$ in the eventual $\alpha$-phase in Fig. 2a).

The mechanism of the $\varepsilon \rightarrow \alpha$ MT is illustrated in Fig. $4 \mathrm{~b}$. Shearing takes place along $[11-20]_{\mathrm{hcp}}$ (top row) or [-1-120 $]_{\mathrm{hcp}}$ (bottom row) in

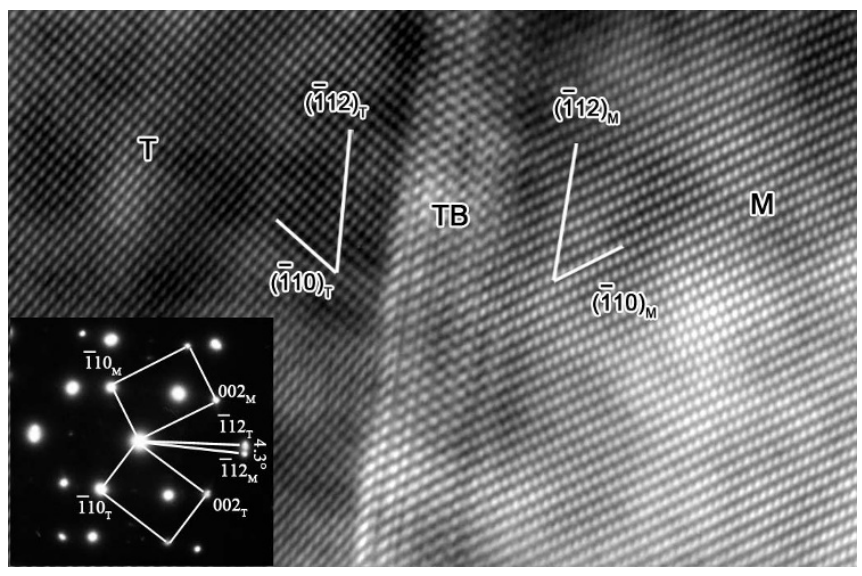

Figure 3 The special character of the needle-like colony's boundary revealed by HRTEM image. This particular example exhibits a $\sim 4.3^{\circ}$ angle between the $(-112)_{\mathrm{T}}$ and the $(-112)_{\mathrm{M}}$ planes, which is also confirmed by the SAED pattern in the inset. 


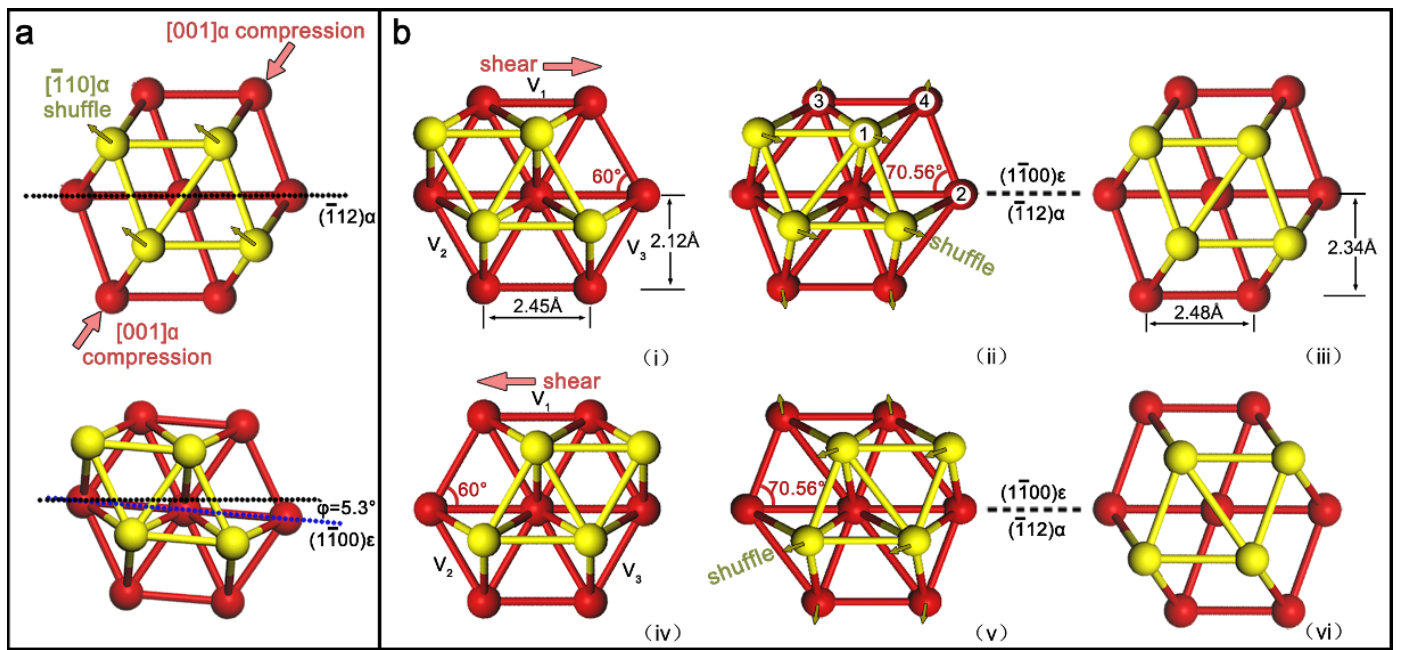

Figure $4 \mid$ Atomic mechanisms of the $\boldsymbol{\alpha} \rightarrow \boldsymbol{\varepsilon} \rightarrow \boldsymbol{\alpha}$ phase transformation. (a) The $\boldsymbol{\alpha} \rightarrow \boldsymbol{\varepsilon}$ phase transformation by compression-shuffle mechanism under shock-loading9. (b) The $\varepsilon \rightarrow \alpha$ phase transformation by shear-shuffle mechanism. The $(110)_{\alpha}$ and $(0001)_{\varepsilon}$ planes are in the plane of the paper. The red and yellow balls with bonds denote two atomic layers in or above the page, respectively. In (a), the bcc structure (top) is compressed along the [001 $]_{\mathrm{bcc}}$ direction by $18.4 \%$, and every other $(110)_{\text {bcc }}$ plane shuffles by $a_{0} /(3 \sqrt{2})$ toward the $[-110]_{\text {bcc }}$ direction (small arrows) to form an hcp structure (bottom). The $[001]_{\mathrm{bcc}}$ compression without lattice expansion in the $[-110]_{\mathrm{bcc}}$ direction results in a tilt angle of $\varphi=5.3^{\circ}$, between the resulting $(1-100)_{\mathrm{hcp}}$ plane and the original $(-112)_{\mathrm{bcc}}$ plane. In (b), shearing takes place either along [11-20 $]_{\mathrm{hcp}}$ (top row) or $[-1-120]_{\mathrm{hcp}}$ (bottom row) in the (1-100) hcp $_{\text {plane, resulting in }}$ bcc lattices with a (-112) twinned relationship (compare iii and vi). Small shuffles (arrows) are required for various types of atoms (labeled 1-4 in the top middle panel). Executing the above shear-shuffle from the three crystallographically equivalent $\{1-100\}_{\text {hcp }}$ planes, three variants $\left(V_{1}\right.$ through $\left.V_{3}\right)$ are possible for the eventual transformation product, resulting in the three sets of $\{112\}_{\mathrm{bcc}}$ twins that retain three-fold symmetry.

the $(0001)_{\text {hcp }}$ plane. Meanwhile, the lattice parameter $c_{\varepsilon}$ expands from 3.93 to $4.05 \AA$ while $a_{\varepsilon}$ increases from 2.45 to $2.48 \AA$, and the $60^{\circ}$ angles on the $(0001)_{\text {hcp }}$ plane change to the $70.56^{\circ}$ on $(112)_{\mathrm{bcc}}$ in the process. Near the shearing atomic row, atoms shuffle with various displacements: e.g., atom 1 through 4 in Fig. 4 b(ii) by $0.832 \AA, 0.032$ $\AA$, $0.4258 \AA$, and $0.454 \AA$, respectively. Shearing in two opposite directions [Figs. $4 \mathrm{~b}$ (i) vs. $4 \mathrm{~b}$ (iv)] is equivalent geometrically but results in two mirror bcc lattices with regard to the $(-112)_{\text {bcc }}$ plane [compare Figs. $4 \mathrm{~b}$ (iii) and $4 \mathrm{~b}(\mathrm{vi})$ ]. Executing the above shear-shuffle from the three crystallographically equivalent $\{1-100\}_{\text {hcp }}$ planes, three variants $\left(V_{1}\right.$ through $V_{3}$ ) are possible for the eventual transformation product, resulting in the three sets of $\{112\}_{\text {bcc }}$ twins that retain three-fold symmetry. This explains how the $\varepsilon \rightarrow \alpha$ reverse transformation leads to the twinned structure inside the needle colonies. What we actually observe under TEM thus matches exactly what one would expect to see in the microstructure from this $\varepsilon \rightarrow \alpha$ MT mechanism.

\section{Discussion}

It should be noted that the shock pressure direction would be along a different orientation for each grain in our polycrystalline sample. In some original $\alpha$-phase grains, the phase transition needles form predominantly along only one $\{112\}_{\text {bcc }}$ plane (Supplementary Fig. S3), rather than along two crystallographically equivalent $\{112\}_{\text {bcc }}$ planes as in Fig. 2. Along the other $\{112\}_{\text {bcc }}$ planes, large Schmid factor activates plastic deformation (deformation twinning without the characters of the three-fold symmetry and the $\varphi \approx 5^{\circ}$ at the twin boundary). However, all the characteristic features of the needle-like regions (transformed regions) in Supplementary Fig. S3 are the same with that in Fig. 2, so the same phase transition mechanism (Fig. 4) is activated irrespective of the varying grain orientations.

Some researchers have predicted that a face-centered cubic (fcc) $\gamma$ Fe phase would occur under shock-loaded iron, based on moleculardynamics simulation or numerical simulations ${ }^{13,21}$. Here we have considered this possibility but excluded the involvement of intermediate $\gamma$-Fe in the phase transition of shock-loaded iron. If the fcc $\gamma$-Fe was the intermediate phase instead of the hcp $\varepsilon$-Fe in the phase transition, the martensite $\alpha$-Fe and the matrix $\gamma$-Fe would have an orientation relationship of $\{111\}_{\mathrm{fcc}} / /\{011\}_{\mathrm{bcc}}{ }^{23,24}$. The $\{011\}_{\mathrm{bcc}}$ planes of the martensite variants (lamellar twins) would present the angle of the $\{111\}_{\mathrm{fcc}}$ planes. The expected angle is $70.5^{\circ}$, which is not observed in our experiments.

We now summarize the entire $\alpha \rightarrow \varepsilon \rightarrow \alpha$ process during shock treatment, using a schematic illustration in Fig. 5. In the original $\alpha$-grain (green in Fig. $5 \mathrm{a}$ ), the $\varepsilon$-needles (blue) form along one or two $\{112\}_{\text {bcc }}$ planes during shock-loading (Fig. 5b), with a characteristic tilt angle at the colony boundaries, resulting from the compression-shuffle mechanism known for the $\alpha \rightarrow \varepsilon$ MT. Afterwards, the needle-like $\varepsilon$-phases transform back to the $\alpha$-phases during unloading: three variants of the $\alpha$-phase emerge inside the needles, inheriting the threefold symmetry from the $\{1-100\}_{\text {hcp }}$ planes of the $\varepsilon$ structure. The shearing and shuffling mechanism involved in the $\varepsilon \rightarrow \alpha$ transformation results in twinned structures, and the final configuration is therefore three $\{112\}<111>$ twin sets (Fig. 5c) with an unusual $60^{\circ}$ impinge angle. These morphological and orientational features observed in the microstructure then serve as a tell-tale sign of the reversible MTs $(\alpha \rightarrow \varepsilon \rightarrow \alpha)$.
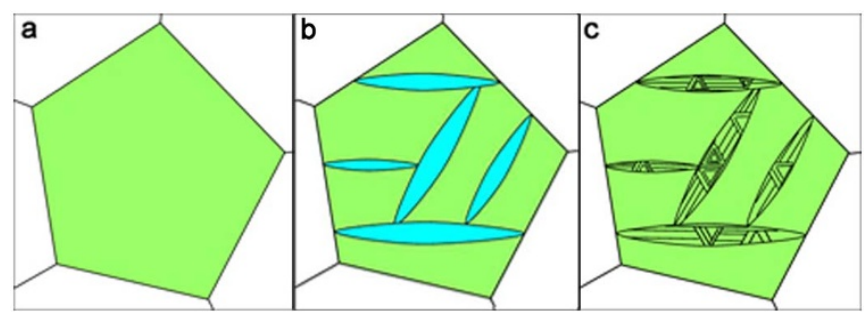

Figure 5 Schematic illustration of the $\alpha \rightarrow \varepsilon \rightarrow \alpha$ phase transformations. (a) The original $\alpha$-grain. (b) The $\alpha \rightarrow \varepsilon$ phase transition under shockloading, forming needle-like $\varepsilon$ regions. (c) The $\varepsilon \rightarrow \alpha$ reverse transition inside the $\varepsilon$-needles upon unloading. Green and blue colors stand for the $\alpha$-phase and the $\varepsilon$-phase, respectively. Black lines are boundaries of the microstructural features. 
Importantly, having identified clear microstructural indicators, the volume fraction transformed, $F$, can now be assessed not only quantitatively (the previously reported estimate under shock-loading had to fit an empirical function to the experimental Hugoniot ${ }^{25}$ ), but also at different locations for a large sample after massive shock treatment. $F$ can be estimated by directly measuring the area of needle-like regions on the EBSD micrographs acquired in scanning electron microscopy (SEM) at a number of locations along the radius in the whole upper surface of the shock-loaded hollow cylinder. As the distance inward from the loading surface increases, the shockloaded pressures decreases, thus $F$ decreases: for example, $54.6 \pm 3.2 \%$ (for the statistical details, see Supplementary Table S2) at $1.2 \mathrm{~mm}$ with $\sim 14.2 \mathrm{GPa}$ (such as Fig. 1d) and $27.1 \pm 2.8 \%$ (see Supplementary Table S3 for details) at $1.9 \mathrm{~mm}$ with $\sim 13.3 \mathrm{GPa}$ (such as Fig. 1c). Such a quantitative measure is very important for profiling and monitoring the shock response of a bulk material, and for validating models and predictions.

In summary, in shock-compressed Fe we have detected and deciphered microstructural fingerprints that can be traced to $\alpha \rightarrow \varepsilon \rightarrow \alpha$ transformation, using postmortem samples under TEM. While there are other in-situ measurements that can detect this phase transition under (local) shock-loading, our method provides more detailed information on the transformation mechanism. More importantly, the evidence we acquired is based on microstructures (characteristic morphologies). The signature features, i.e., the needle-type regions and their boundary characteristics as well as the angle between the twinned lamella inside, are uniquely revealing of the MTs involved. From them we can tell apart the transformed areas from those that have not experienced the MT. This has enabled a quantitative evaluation of the volume percentage of the transient $\varepsilon$-phase formed at different locations and pressures under a complex shock-loading condition in a polycrystalline sample. In particular, our postmortem "microstructure" approach has advantages in removing many difficulties and limitations associated with the use of relative intensities of in-situ X-ray diffraction peaks (e.g., signal to noise ratio, average over the probed thickness, limited sample shape/size/thickness and shock-loading conditions). The method of crystallographic analysis in the present work reproduced the formation process of the lamellar twins in the shocked iron. The same method may also be useful for inferring the origin of the Neumann bands which are mechanical twin lamellae formed under extraterrestrial collisions in bcc structured kamacite phase in meteorites ${ }^{26,27}$.

\section{Methods}

Experimental setup. The experimental setup is schematically shown in Supplementary Fig. S4. Polycrystalline Fe with a purity of $99.90 \%$ was used to fabricate a $60 \mathrm{~mm}$ high hollow cylinder with a $42.5 \mathrm{~mm}$ outer radius (R) and a $22.5 \mathrm{~mm}$ inner radius (r) (Supplementary Fig. S4a). The outside surface of the cylinder was covered with a $2.5 \mathrm{~mm}$ organic glass buffer layer followed by a $15 \mathrm{~mm}$ PETN explosive layer (as shown in Supplementary Fig. S4c). The entire explosive was detonated nearly at the same time by the detonating system illustrated in Supplementary Fig. S4c. The shock wave proceeds in the radial direction of the cylinder labeled by the purple arrows in Supplementary Fig. S4b. A VISAR equipment located in the center of the hollow cylinder (shown in Supplementary Fig. S4c) was used to record the inner free surface velocity (Supplementary Fig. S5). The structural evolution was investigated using the EBSD technique on a JEOL SEM 6700 with a field emission gun and TEM (JEM2010 and FEI Tecnai F30).

Pressure calculations. The experimental setup cannot record the velocity for every point inside the sample. So the pressure history in the sample was calculated using a mixed phase model proposed by $\mathrm{Hayes}^{28}$ and Andrews ${ }^{29}$ based on a 2-D

hydrodynamic code. Also, Boettger's model on metastable surfaces for forward and reverse phase transitions in pure iron and the parameters from his article ${ }^{25}$ were used in the calculation. According to the calculation for every point along the radial direction in the sample, with increasing distance inward from the loading surface, the peak pressure decreases. At every point the explosively driven shockwaves rise rapidly in pressure and then release at a slower timescale. The peak pressure of the location near the cylinder outside/shock loading surface is $\sim 16.4 \mathrm{GPa}$. To compare with the experimental observations, the calculated pressure histories of the points at 4.6, 1.9, and $1.2 \mathrm{~mm}$ inward from the loading surface are shown in Supplementary Fig. S6a. The peak pressures at the points $4.6,1.9$, and $1.2 \mathrm{~mm}$ are $\sim 12.7, \sim 13.3$ and $\sim 14.2 \mathrm{GPa}$, respectively. The inner free surface velocity curves from the calculation and experiment are shown in Supplementary Fig. S6b, which are in good agreement with each other.

1. Saxena, S. K. \& Dubrovinsky, L. S. Iron phases at high pressures and temperatures: Phase transition and melting. Am. Mineral. 85, 372-375 (2000).

2. Walsh, J. M. Metal Equation of State from Shock-Wave Studies. Bull. Am. Phys Soc. 29, 28 (1954).

3. Bancroft, D., Peterson, E. L. \& Minshall, S. Polymorphism of iron at high pressure. J. Appl. Phys. 27, 291-298 (1956).

4. Minshall, S. Investigation of a polymorphic transition in iron at $130 \mathrm{~kb}$. Phys. Rev. 98, 271 (1955).

5. Duff, R. E. \& Minshall, F. S. Investigation of a shock-induced transition in bismuth. Phys. Rev. 108, 1207-1212 (1957).

6. Barker, L. M. \& Hollenbach, R. E. Shock wave study of the $\alpha-\varepsilon$ phase transition in iron. J. Appl. Phys. 45, 4872-4887 (1974).

7. de Rességuier, T. \& Hallouin, M. Effects of the $\alpha-\varepsilon$ phase transition on wave propagation and spallation in laser shock-loaded iron. Phys. Rev. B 77, 174107 (2008).

8. Kalantar, D. H. et al. Direct observation of the $\alpha-\varepsilon$ transition in shock-compressed iron via nanosecond X-ray diffraction. Phys. Rev. Lett. 95, 075502 (2005).

9. Hawreliak, J. et al. Analysis of the $\mathrm{x}$-ray diffraction signal for the $\alpha-\varepsilon$ transition in shock-compressed iron: Simulation and experiment. Phys. Rev. B 74, 184107 (2006).

10. Hawreliak, J. A. et al. In situ x-ray diffraction measurements of the c/a ratio in the high-pressure $\varepsilon$ phase of shock-compressed polycrystalline iron. Phys. Rev. B 83, 144114 (2011).

11. Yaakobi, B. et al. EXAFS measurement of iron bcc-to-hcp phase transformation in nanosecond-laser shocks. Phys. Rev. Lett. 95, 075501 (2005).

12. Kadau, K., Germann, T. C., Lomdahl, P. S. \& Holian, B. L. Microscopic view of structural phase transitions induced by shock waves. Science 296, 1681-1684 (2002).

13. Kadau, K., Germann, T. C., Lomdahl, P. S. \& Holian, B. L. Atomistic simulations of shock-induced transformations and their orientation dependence in bcc Fe single crystals. Phys. Rev. B 72, 064120 (2005).

14. Kadau, K. et al. Shock waves in polycrystalline iron. Phys. Rev. Lett. 98, 135701 (2007).

15. Guo, Y. F., Wang, Y. S., Zhao, D. L. \& Wu, W. P. Mechanisms of martensitic phase transformations in body-centered cubic structural metals and alloys: Molecular dynamics simulations. Acta Mater. 55, 6634-6641 (2007).

16. Dobromyslov, A. V., Kozlov, E. A. \& Taluts, N. I. High-strain-rate deformation of armco iron induced by spherical and quasi-spherical converging shock waves and the mechanism of the $\alpha-\varepsilon$ transformation. Phys. Met. and Metal. 106, 531-541 (2008).

17. Dougherty, L. M. et al. Rare twin linked to high-pressure phase transition in iron. Scr. Mater. 60, 772-775 (2009).

18. Sano, T., Mori, H., Ohmura, E. \& Miyamoto, I. Femtosecond laser quenching of the $\varepsilon$ phase of iron. Appl. Phys. Lett. 83, 3498-3500 (2003).

19. Krauss, G. \& Marder, A. R. The morphology of martensite in iron alloys. Metall. Trans. 2, 2343-2357 (1971).

20. Zhang, W., Zhao, W. S., Li, D. X. \& Sui, M. L. Martensitic transformation from $\alpha-$ Ti to $\beta$-Ti on rapid heating. Appl. Phys. Lett. 84, 4872-4874 (2004).

21. Biichikov, S. A. et al. In Shock Compression of Condensed Matter-2007, Pts 1 and 2 Vol. 955 (eds Elert, M., Furnish, M. D., Chau, R., Holmes, N. \& Nguyen, J.) 251-254 (Waikoloa, Hawaii, 2007).

22. Wang, F. M. \& Ingalls, R. Iron bcc-hcp transition: Local structure from x-rayabsorption fine structure. Phys. Rev. B 57, 5647-5654 (1998).

23. Kurdjumov, G. V. \& Sachs, G. Over the mechanisms of steel hardening. Z. Phys 64, 325-343 (1930)

24. Nishiyama, Z. X-ray Investigation of the Mechanism of the Transformation from Face-centered Cubic Lattice to Body-centered Cubic. Sci. Rep. Tohoku Univ. 23, 637-664 (1934)

25. Boettger, J. C. \& Wallace, D. C. Metastability and dynamics of the shock-induced phase transition in iron. Phys. Rev. B 55, 2840-2849 (1997).

26. He, Y., Godet, S., Jacques, P. J. \& Jonas, J. J. Crystallographic relations between face- and body-centred cubic crystals formed under near-equilibrium conditions: Observations from the Gibeon meteorite. Acta Mater. 54, 1323-1334 (2006).

27. Teshima, J., Wasserburg, G. J., El Goresy, A. \& Chen, J. H. A comparative petrologic study of iron meteorites with ${ }^{107} \mathrm{Ag}$ anomalies. Geochim. Cosmochim. Acta 50, 2073-2087 (1986).

28. Hayes, D. Wave propagation in a condensed medium with $\mathrm{N}$ transforming phases: Application to solid-I-solid-II-liquid bismuth. J. Appl. Phys. 46, 3438-3443 (1975)

29. Andrews, D. J. Calculation of mixed phases in continuum mechanics. J. Comput. Phys. 7, 310-326 (1971).

\section{Acknowledgments}

This work was supported by the National Natural Science Foundation of China (NSFC) under Grant No. 10776032, and the Cheung Kong Scholars Program of China. We are also indebted to Prof. Y. Ji of BJUT for assistance in the EBSD experiments. 


\section{Author contributions}

S.J.W. and Q.H.L. carried out the TEM experiments. Y.T.C., Q.Z.L. and H.B.H. carried out the shock experiments. X.Y.P. performed the pressure calculations. M.L.S. and E.M. and S.J.W. wrote the paper. All authors contributed to the discussions.

\section{Additional information}

Supplementary information accompanies this paper at http://www.nature.com/ scientificreports
Competing financial interests: The authors declare no competing financial interests.

License: This work is licensed under a Creative Commons

Attribution-NonCommercial-NoDerivs 3.0 Unported License. To view a copy of this license, visit http://creativecommons.org/licenses/by-nc-nd/3.0/

How to cite this article: Wang, S.J. et al. Microstructural fingerprints of phase transitions in shock-loaded iron. Sci. Rep. 3, 1086; DOI:10.1038/srep01086 (2013). 